1 Running head: Balance in older patients with type 2 diabetes

2

3 Validity and Relative Ability of Four Balance Tests to Identify Fall Status of Older Adults with Type 2 Diabetes

5 Alda Marques PT, PhD 1,2, Alexandre Silva PT, MSc 1, Ana Oliveira PT, MSc 1,2, Joana Cruz

6 PT, PhD 1,2, Ana Machado PT 1,2, Cristina Jácome PT, PhD 1

71 Lab 3R - Respiratory Research and Rehabilitation Laboratory, School of Health Sciences,

8 University of Aveiro (ESSUA), Aveiro, Portugal

92 Institute for Research in Biomedicine (iBiMED), University of Aveiro, Aveiro, Portugal

11 Corresponding author: Alda Marques, PT, PhD, Senior Lecturer, Lab 3R - Respiratory Research 12 and Rehabilitation Laboratory, School of Health Sciences, University of Aveiro (ESSUA), Agras do Crasto - Campus Universitário de Santiago, Edifício 30, 3810-193 Aveiro, Portugal. Telephone number: 00351234372 462. Fax number: 00351234401 597. Email: amarques@ua.pt

Conflicts of Interest and Source of Funding: This work was also partially supported by FEDER

17 through COMPETE and FCT under project UID/BIM/04501/2013 and by National Funds through FCT, in the context of the projects UID/CEC/00127/2013 and Incentivo/EEI/UI0127/2014. The authors have no conflicts of interest to declare. 
ABSTRACT

Background and Purpose: The Berg Balance Scale (BBS), the Balance Evaluation Systems Test (BESTest), the Mini-BESTest and the Brief-BESTest are useful tests to assess balance, however their psychometric properties have not been studied well in older adults with type 2 diabetes. This study compared the validity and relative ability of the Berg Balance Scale (BBS), Balance Evaluation Systems Test (BESTest), Mini-BESTest and Brief-BESTest to identify fall status in older adults with type 2 diabetes (T2D).

Methods: This study was a cross-sectional design. Sixty-six older adults with T2D ( $75 \pm 7.6$ years) were included and asked to report the number of falls during the previous 12 months and to complete the Activity-specific Balance Confidence (ABC) Scale. The BBS and the BESTest were administered, and the Mini-BESTest and Brief-BESTest scores were computed based on the BESTest performance. Receiver operating characteristics were used to assess the ability of each balance test to differentiate between participants with and without a history of falls.

Results: The 4 balance tests were able to identify fall status (areas under the curve $[A U C]=0.74$ $0.76)$, with similar sensitivity (60-67\%) and specificity (71-76\%).

Conclusions: The 4 balance tests were able to differentiate between older adults with T2D with and without a history of falls. As BBS and BESTest require longer application time, the BriefBESTest may be an appropriate choice to use in clinical practice to detect fall risk. 
40 Over the past 3 decades, the number of people with diabetes has more than doubled. ${ }^{1}$ The World

41 Health Organization projects that diabetes will be the $7^{\text {th }}$ leading cause of death in $2030 .{ }^{2}$ The disease represents a tremendous current challenge for health care systems and societies. Diabetes is a chronic disease that occurs either when the pancreas does not produce enough insulin or when the body cannot effectively use the insulin it produces. ${ }^{3}$ Type 2 diabetes (T2D) is the most common type of the disease, representing $90 \%$ of patients with diabetes. ${ }^{3}$ Usually T2D develops in older adults aged 65 and 75 years old and is related to obesity, lack of physical activity and unhealthy diets. ${ }^{4}$

Microvascular complications (e.g., retinopathy, nephropathy and neuropathy) and increased risk of macrovascular complications (e.g., ischemic heart disease, stroke and peripheral vascular disease) are characteristic of this chronic health condition. ${ }^{3}$ Evidence shows that older adults with over 25 years history of T2D have distal sensory polyneuropathy, with sensitivity characteristic of diabetic foot, which reduces the ability to control postural reactions and increase the risk of falling. ${ }^{5}$ Both $\mathrm{T} 2 \mathrm{D}$ and falls are well-known contributors for significant morbidity, diminished quality of life and reduced life expectancy ${ }^{3}$ and thus, it is imperative to assess risk of falling in this population.

To assess balance and identify risk of falling, a number of balance tests have been developed. However, as pointed out by Duncan et al, to predict falls these tests should be: i) theoretically grounded in examining the systems controlling balance, ii) accurate in their ability to predict falls, and iii) feasible and practical for clinical use. ${ }^{6}$

The Berg Balance Scale (BBS) has been one of the most commonly used tests to identify balance limitations. ${ }^{7}$ The BBS has been used to characterize balance in older adults with T2D. ${ }^{8}$ It is an inexpensive test, easy to administer (approximately 20 minutes), ${ }^{7}$ and with the ability to predict risk of falling in community-dwelling older adults. ${ }^{9}$ However, the BBS has limitations such as a ceiling effect and the redundancy of categories due to the rating scale. ${ }^{10}$ These limitations are important to consider when assessing patients with mild motor and neurological impairments, who may not be identified as at risk of falling and therefore less likely to be offered the appropriate intervention. ${ }^{10}$ 

components contributing to dysfunctional balance. ${ }^{11}$ This test has been used to assess balance in older adults with diabetes. ${ }^{12}$ However, its clinical feasibility is limited, due to the time required to complete all 36 items (approximately 20-30 minutes). ${ }^{13}$ To address these limitations, shortened versions, the Mini-BESTest and the Brief-BESTest, were developed. The Mini-BESTest includes important aspects of dynamic balance control, reflecting balance challenges during activities of daily living (administration takes approximately 15 minutes). ${ }^{14}$ This test has been shown to be useful for assessing balance in patients with other chronic health conditions. ${ }^{14,15}$ In contrast to the Mini-BESTest, the Brief BESTest contains items that assess all balance systems originally outlined by the original BESTest. ${ }^{13}$ The Brief-BESTest requires less administration time (approximately 10 minutes) and less equipment than the Mini-BESTest, which could favor its clinical use. ${ }^{13}$

The use of an excessive number of balance tests has hampered consistent clinical practice and recently an expert panel recommended that at a minimum, either the BBS or MiniBESTest, should be used when measuring balance in adult populations. ${ }^{16}$ Given the specific clinical characteristics of older adults with T2D and the different balance tests available, it is crucial that clinicians know which are the most useful and sensitive tests to identify older adults with T2D at risk of falling. However, to the authors' knowledge, the utility of the 4 balance tests described above have not yet been analyzed in this specific population. Therefore, the aim of this study was to compare the validity and relative ability of the BBS, BESTest, Mini-BESTest and Brief-BESTest to identify fall status in older adults with T2D. METHODS

Study Design and Participants

A cross-sectional study with a sample of older adults with T2D was conducted from November 2014 to February 2015 in the central region of Portugal. Participants were recruited from 3 primary care centers (the first point of health consultation in the National Health Service) and 5 daycare centers (i.e., centers that offer a range of recreational, cultural, educational, health and social support services to older adults). Ethical approval was obtained from the Ethics Committee (238/10-2014). Inclusion criteria were a diagnosis of T2D according to the World Health 
study and voluntarily consent to participate. Older adults were excluded if they had severe musculoskeletal, neurological, cardiovascular or psychiatric disorders; used medications that may increase the risk of falls; and if they had limited ability to walk and/or severe auditory/visual impairments. Older adults were identified and screened by the general practitioners of the primary care/day care centers involved. Researchers then contacted eligible older adults to explain the purpose of the study and ask about their willingness to participate. When older adults with T2D agreed to participate, an appointment was scheduled at the primary care center or daycare center more convenient to the participant. Written informed consent was obtained prior to data collection.

\section{Data Collection Procedures}

Two qualified physical therapists, with at least 4 years of experience in working with older adults with chronic health conditions, performed all the assessments. First, sociodemographic (gender, age and occupation) and anthropometric (height, weight, body mass index) data were collected. Second, participants were asked about comorbidities (e.g., hypertension and hyperlipidemia) and falls history. Patients were provided with a clear definition of falls ("an event when you find yourself unintentionally on the ground, floor or lower level" $)^{17}$ and asked about their history of falls using 2 standardized questions (1."Have you had any falls in the last 12 months?" and, if yes, 2. "How many times did you fall down in the last 12 months?"). ${ }^{18}$ The reference to the last 12 months has been recommended by international guidelines ${ }^{19}$ and is more commonly used as an outcome measure..$^{20}$ Then, the Activities Specific Balance Confidence (ABC) Scale was used to quantify how confident the participant feels that he or she will not lose balance while performing 16 activities of daily living. ${ }^{21}$ Participants received explanations about the aim of the $A B C$ scale and were asked to complete it by themselves. For participants who were unable to read, ABC Scale was interviewer-administered.

Lastly, the BBS and the BESTest were performed and participants were encouraged to rest, as needed. To ensure competency in applying the balance tests, prior to the data collection period, the 2 physical therapists read the testing procedures and practiced administering between them. Excellent inter-rater reliability (ICC from 0.85 to 0.94 ) has been previously reported for these tests. $^{22,23}$ For each item of the BBS or BESTest, the physical therapist read the standardized instructions and demonstrated the task. The participant then performed the task with close supervision. Each task was scored immediately after completion. Mini-BESTest and Brief- 
BESTest scores were computed based on the performance of the BESTest tasks. A custom designed worksheet was used to simultaneously record the BESTest and Mini-BESTest item scores. Brief-BESTest scores were extracted from the relevant subset of BESTest items.

Measures

The BBS is composed of 14 items that require participants to maintain positions of varying difficulty and perform specific tasks, such as standing and sitting unsupported, transfers (sit to stand and stand to sit), turn to look over shoulders, pick up an object from the floor, turn $360^{\circ}$ and place alternate feet on a stool. ${ }^{7}$ Scoring is based on the participant's ability to perform the 14 tasks independently and/or meet certain time or distance requirements. Each item was scored on a 5-point ordinal scale ranging from 0 (unable to perform) to 4 (normal performance), so that the total score ranged from 0 to $56 .^{7}$

The BESTest consists of 36 items that evaluate 6 subsystems of balance control: 1) biomechanical constraints, 2) stability limits/verticality, 3) anticipatory postural adjustments, 4) postural responses, 5) sensory orientation and 6) gait stability. ${ }^{11}$ Each item is graded on a 4-point ordinal scale from 0 (unable to perform) to 3 (normal performance) as judged by time or performance criteria. Each subsystem category comprises $20 \%$ of the total balance score. The BESTest total score is a sum of all the individual items, with a maximum of 108 (higher scores indicate better balance). ${ }^{11}$

The Mini-BESTest is a shortened version of BESTest that includes only 14 of the original 36 items, that focus on dynamic balance, specifically anticipatory transitions, postural responses, sensory orientation, and dynamic gait. ${ }^{14}$ Two of the 14 items were assessed bilaterally, but only the lower score was used for the total score. Although all items of the Mini-BESTest are included in the BESTest, the grading criteria is different. Each item is scored from 0 (severe balance limitation) to 2 (no balance limitation) and the maximum possible score is 28 points. ${ }^{14}$ Higher scores indicate better balance performance.

The Brief-BESTest is an 8-item revised version of the BESTest designed to improve the clinical utility and to preserve the construct validity of BESTest. ${ }^{13}$ Items from each of the BESTest subsystems were selected to develop the Brief-BESTest based on the highest item correlation coefficients with each subsystem. ${ }^{13}$ Each item is scored from 0 (representing severe limitation) to 

scores indicate better balance performance.

Descriptive statistics were used to describe the sociodemographic, anthropometric and clinical characteristics of the sample and the scores on the BBS, BESTest, Mini-BESTest and BriefBESTest. Characteristics were compared between participants with and without a history of falls using independent t-tests for normally distributed data, Mann-Whitney U-tests for non-normally distributed and Chi-square tests for categorical data. Individuals with a history of falls were defined as those who reported at least one fall during the past year; individuals without a history of falls were defined as those who reported no falls during the past year. Spearman's correlation (rho) was used to examine the relationship among balance tests (concurrent validity) and between each balance test and the $\mathrm{ABC}$ Scale (convergent validity).

Receiver operating characteristic curves were used to assess the ability of each balance test to differentiate between participants with and without a history of falls. Area under the curves (AUC), together with the $95 \%$ confidence intervals, were determined and AUC interpreted as follows: $A \cup C=0.5$ no discrimination; $0.7 \leq A \cup C<0.8$ acceptable discrimination; $0.8 \leq A \cup C<0.9$ excellent discrimination and $A U C \geq 0.9$ outstanding discrimination. ${ }^{24}$ The AUC is the probability of correctly identifying an older adult with T2D who has a history of falls in randomly selected pairs of older adults who have and do not have a history of falling. ${ }^{25}$ The cutoff for each balance test was chosen as the point where the sensitivity and specificity were simultaneously maximized. The positive and negative likelihood ratios (LR+ and LR-) were also computed.26 Corporation, Armonk, NY, USA) and plots created using GraphPad Prism version 5.01 (GraphPad Software, Inc., La Jolla, CA, USA). The level of significance considered was 0.05 .

\section{RESULTS}

Participants' Characteristics

A total of 80 older adults with T2D were contacted; however, 9 did not want to participate and 5 did not complete the assessment. Therefore, 66 participants (38 females) were enrolled. The mean age was $75 \pm 7.6$ years old and the mean body mass index was $29.2 \pm 4.4 \mathrm{Kg} / \mathrm{m}^{2}$. Almost all 
participants (90.9\%) were retired and $37.9 \%$ reported a history of falling. Participants'

187

188

189

190

191

192

193

194

195

196

197

198

199

200

201

202

203

204

205

206

207

208

209

210

211

212

213

214

215 characteristics are presented in Table 1.

(table 1)

Validity

The 4 balance tests were strongly correlated, with Spearman's correlations coefficients from 0.85 to $0.91(p<0.001)$. The ABC scale was significantly correlated with all the balance tests (rho from 0.62 to $0.70 ; p<0.001$ ) (Figure 1).

(figure 1)

Ability to Identify Fall Status

Table 2 presents the results from the ROC analysis. The AUCs for the 4 balance tests had a satisfactory performance with values ranging between 0.74 and 0.76 , with similar confidence intervals. Cutoff points were identified: 50.5 points for the BBS (sensitivity=64\%; specificity=76\%); 81 points for the BESTest (sensitivity=68\%; specificity=71\%); 20.5 points for the Mini-BESTest (sensitivity=60\%; specificity=71\%); and 15.5 points for the Brief-BESTest (sensitivity=67\%; specificity=71\%) (Figure 2). The Mini-BESTest had the lower positive likelihood ratio ( $(\mathrm{R}+=2.05)$ and the higher negative likelihood ratio $(\mathrm{LR}-=0.57)($ Table 2$)$.

(table 2 and figure 2)

\section{DISCUSSION}

To our knowledge, this is the first study to compare the validity and relative ability of BBS, BESTest, Mini-BESTest and Brief-BESTest to identify fall status in older adults with T2D. This study showed that the 4 balance tests presented acceptable ability to differentiate between older adults with T2D with and without a history of falls with similar sensitivity/specificity.

A total of $37.9 \%$ of older adults with T2D reported a history of falling. This prevalence is similar to previous studies (35-41\%), where older adults with T2D with equivalent mean ages and gender ratios were included. $8,27,28$ In healthy older adults, similar, but slightly lower, prevalences have been described (12.1-33\%). 27,29,30 This finding corroborates previous research demonstrating that older adults with T2D present higher incidence of falls compared to healthy older people..$^{27,28}$

The BBS, BESTest, Mini-BESTest and Brief-BESTest have shown to be valid in a variety of clinical populations. ${ }^{10,31,32}$ In older adults with T2D, good concurrent and convergent validity 
were also found. All 4 balance tests had an acceptable ability to differentiate between participants with and without a history of falls (AUCs>0.70), with similar confidence intervals. The cut-offs other populations, BBS (48.5-52.5 $5^{15,22,23}$ points), BESTEST (69-82 points $\left.{ }^{6,22,23}\right)$, Mini-BESTest $\left(19.5-21.5^{6,15,22,23}\right.$ points) and Brief-BESTest (11-16.5 $5^{6,22,23}$ points). Moreover, the cutoff points identified demonstrated similar sensitivities (60-68\%) and specificities (71-76\%) between each balance test. Previous studies have found slightly higher sensitivities (64-89\%) and specificities $(65-84 \%), 6,15,22,23$ which may be partially related with larger sample sizes included. The cutoff points identified can be used by clinicians to detect older adults with T2D at risk of falling and to implement preventive interventions. However, when analyzing the likelihood ratios, the MiniBESTest was the test with the lower performance. These results are important for clinical practice since they suggest that clinicians may more confidently rely on the BBS, BESTest and BriefBESTest to identify older adults with T2D at risk of falling. In addition, as these 3 tests have similar discrimination ability, the Brief-BESTest may be preferable when time or resources to perform balance assessment are limited. At this point in time, however, it is not known whether the differences in the ability to identify fall status among balance tests are clinically meaningful. For example, neuropathy could make this population more likely to perform poorer in one or more domains of the balance tests as compared to other populations with chronic health conditions. Future studies could identify the items from BBS and BESTest with highest predictive ability to identify fall status in patients with T2D. This would be valuable in the design of tailored interventions. Furthermore, as the Brief BESTest is a less time consuming test and has one item from each domain, it would be interesting to study if those items adequately identify balance impairments in the population with T2D.

Our study has some limitations. First, this study included only older adults with T2D, so findings cannot be generalized for people with T2D at all ages. Future studies may replicate the study in larger samples of younger people and report results per age decade. A second limitation is that participants were classified with a history of falls in the last year based on self-report, however, they may have forgotten or under-reported their falls. ${ }^{33,34}$ Recent studies used selfreported falls in the last 6 months, ${ }^{6,35}$ this approach may be also of interest to use in future research. Third, balance tests were administered by the same physical therapists who assessed 
fall history. This could have influenced the results. In order to maintain blinding with respect to fall history, future studies should assess fall history after the administration of balances tests ${ }^{6}$ or have different raters assessing these components. Fourth, BESTest, Mini-BEST and Brief-BESTest were scored concurrently based on a single performance. Considering the length of the BESTest, it is possible that inter-item influences may have occurred. Future studies should assess the ability to identify fall status of the Mini-BESTest and the Brief-BESTest when performed separately from

252 the BESTest. Finally, as this was a cross-sectional study, the ability of the balance tests to identify fall status was only possible to be analyzed retrospectively. Longitudinal studies should be conducted in order to assess the prospectively ability of these tests in identifying older adults with recurrent falls.

\section{CONCLUSIONS}

257 BBS, BESTest, Mini-BESTest and Brief-BESTest were able to differentiate between older adults with T2D with and without a history of falls. Cutoff points were identified: 50.5 points for the BBS; 81 points for the BESTest; 20.5 points for the Mini-BESTest; and 15.5 points for the BriefBESTest. As BBS and BESTest require longer application time and the Mini-BESTest had the lowest performance, the Brief-BESTest may be an appropriate choice to use in clinical practice to detect fall risk in patients with T2D. It is believed that these findings will help clinicians to assess balance in older adults with T2D and will inform on whom to prioritise intervention. 
Acknowledgements: The authors would like to acknowledge the participants in this study.

267

\section{REFERENCES}

1. Chen L, Magliano DJ, Zimmet PZ. The worldwide epidemiology of type 2 diabetes mellitus--present and future perspectives. Nat Rev Endocrinol. 2012;8(4):228-236.

2. Mathers CD, Loncar D. Projections of global mortality and burden of disease from 2002

3. World Health Organization. Definition and diagnosis of diabetes mellitus and intermediate

7. Berg KO, Maki BE, Williams Jl, et al. Clinical and laboratory measures of postural balance in an elderly population. Arch Phys Med Rehabil. 1992;73(11):1073-1080.

8. Cordeiro RC, Jardim JR, Perracini MR, et al. Factors associated with functional balance and mobility among elderly diabetic outpatients. Arq Bras Endocrinol Metab. 2009;53:834-843.

9. Lajoie $\mathrm{Y}$, Gallagher SP. Predicting falls within the elderly community: comparison of postural sway, reaction time, the Berg balance scale and the Activities-specific Balance Confidence $(\mathrm{ABC})$ scale for comparing fallers and non-fallers. Arch Gerontol Geriatr. 2004;38(1):11-26.

10. Godi M, Franchignoni F, Caligari M, et al. Comparison of reliability, validity, and responsiveness of the mini-BESTest and Berg Balance Scale in patients with balance disorders. Phys Ther. 2013;93(2):158-167. 
11. Horak FB, Wrisley DM, Frank J. The Balance Evaluation Systems Test (BESTest) to differentiate balance deficits. Phys Ther. 2009;89(5):484-498.

12. Riddhi D, Ramesh D. Comparison of balance in elderly with and without diabetes using Balance Evaluation Systems Test (BESTest). Indian J Physiother Occup Ther.

13. Padgett PK, Jacobs JV, Kasser SL. Is the BESTest at its best? A suggested brief version

17. Biderman A, Cwikel J, Fried A, et al. Depression and falls among community dwelling elderly people: a search for common risk factors. J Epidemiol Community Health. 2002;56(8):631-636.

18. Zhang F, Ferrucci L, Culham E, et al. Performance on five times sit-to-stand task as a predictor of subsequent falls and disability in older persons. J Aging Health. 2013;25(3):478-492.

19. National Institute for Health and Care Excellence. Falls in older people: assessing risk and prevention. 2013; Available from: nice.org.uk/guidance/cg161.

20. Gillespie LD, Robertson MC, Gillespie WJ, et al. Interventions for preventing falls in older people living in the community. Cochrane Database Syst Rev. 2009(2):CD007146.

21. Branco PS. Validation of the portuguese version of the "Activities-specific Balance Confidence Scale". RSPMFR. 2010;19(2):20-25. 
22. Jácome C, Cruz J, Oliveira A, et al. Validity, Reliability, and Ability to Identify Fall Status of the Berg Balance Scale, BESTest, Mini-BESTest, and Brief-BESTest in Patients With COPD. Phys Ther. 2016; in press.

23. Marques A, Almeida S, Carvalho J, et al. Reliability, validity and ability to identify fall status of the BESTest, Mini-BESTest and Brief-BESTest in older people living in the community. Arch Phys Med Rehabil. 2016;in press.

24. Hosmer DW, Lemeshow S. Applied logistic regression. 2nd ed. New York: Wiley; 2000.

25. Greiner M, Pfeiffer D, Smith RD. Principles and practical application of the receiveroperating characteristic analysis for diagnostic tests. Prev Vet Med. 2000;45(1-2):23-41.

26. Deeks JJ, Altman DG. Diagnostic tests 4: likelihood ratios. BMJ. 2004;329(7458):168169.

27. Chiba $Y$, Kimbara $Y$, Kodera R, et al. Risk factors associated with falls in elderly patients with type 2 diabetes. J Diabetes Complications. 2015;29(7):898-902.

28. Maurer MS, Burcham J, Cheng H. Diabetes mellitus is associated with an increased risk of falls in elderly residents of a long-term care facility. J Gerontol A Biol Sci Med Sci. 2005;60(9):1157-1162.

29. O'Hoski S, Sibley KM, Brooks D, et al. Construct validity of the BESTest, mini-BESTest and briefBESTest in adults aged 50 years and older. Gait Posture. 2015;42(3):301-305.

30. Schumacher J, Pientka L, Trampisch U, et al. The prevalence of falls in adults aged 40 years or older in an urban, German population. Results from a telephone survey. $Z$ Gerontol Geriatr. 2014;47(2):141-146.

31. Tsang CS, Liao LR, Chung RC, et al. Psychometric properties of the Mini-Balance Evaluation Systems Test (Mini-BESTest) in community-dwelling individuals with chronic stroke. Phys Ther. 2013;93(8):1102-1115.

32. Huang $\mathrm{MH}$, Miller $\mathrm{K}$, Smith $\mathrm{K}$, et al. Reliability, validity, and minimal detectable change of Balance Evaluation Systems Test and its short versions in older cancer survivors: a pilot study. J Geriatr Phys Ther. 2015;39(2):58-63.

33. Day L, Fildes B, Gordon I, et al. Randomised factorial trial of falls prevention among older people living in their own homes. BMJ. 2002;325(7356):128. 
354

355

356

357

358

359

360

361

34. Bergland A, Pettersen AM, Laake K. Falls reported among elderly Norwegians living at home. Physiother Res Int. 1998;3(3):164-174.

35. Leddy AL, Crowner BE, Earhart GM. Utility of the Mini-BESTest, BESTest, and BESTest sections for balance assessments in individuals with Parkinson disease. J Neurol Phys Ther. 2011;35(2):90-97. 
Table 1. Participants' characteristics.

\begin{tabular}{|c|c|c|c|c|}
\hline Characteristics & Total $(n=66)$ & $\begin{array}{l}\text { Without a } \\
\text { history of } \\
\text { falls ( } n=41 \text { ) }\end{array}$ & $\begin{array}{l}\text { With a history } \\
\text { of falls }(n=25)\end{array}$ & p-value \\
\hline Female & $38(57.6 \%)$ & $22(53.7 \%)$ & $16(64 \%)$ & .451 \\
\hline Age (years) & $75 \pm 7.6$ & $72.7 \pm 7.1$ & $78.6 \pm 6.9$ & .002 \\
\hline BMI $\left(\mathrm{Kg} / \mathrm{m}^{2}\right)$ & $29.2 \pm 4.4$ & $29 \pm 4.2$ & $29.7 \pm 4.9$ & .529 \\
\hline \multicolumn{5}{|l|}{ Current occupation } \\
\hline Retired & $60(90.9 \%)$ & $36(87.8 \%)$ & $23(92 \%)$ & .366 \\
\hline Employed & $6(9.1 \%)$ & $5(12.2 \%)$ & $2(8 \%)$ & \\
\hline Comorbidities, M[IQR] & $2[1,3]$ & $2[1,2]$ & $1[1,3]$ & .892 \\
\hline ABC scale & $74.5 \pm 25.2$ & $82.2 \pm 18.7$ & $61.9 \pm 29.6$ & .004 \\
\hline BBS & $47.1 \pm 10.4$ & $50.8 \pm 6.3$ & $40.9 \pm 12.9$ & .001 \\
\hline BESTest & $75.7 \pm 18.4$ & $81.2 \pm 14.2$ & $66.7 \pm 20.9$ & .004 \\
\hline Mini-BESTest & $19.6 \pm 6.5$ & $21.8 \pm 4.9$ & $16.2 \pm 7.5$ & .002 \\
\hline Brief-BESTest & $14.3 \pm 6.7$ & $16.5 \pm 5.3$ & $10.6 \pm 7.2$ & .001 \\
\hline
\end{tabular}

365

Note: values show mean \pm standard deviation or $\mathrm{n}(\%)$ unless otherwise indicated. Abbreviations:

ABC, Activities specific Balance Confidence Scale; BMI, Body Mass Index; BBS, Berg Balance

Scale; BESTest, Balance Evaluation Systems Test; M, median; IQR, interquartile range. 
Table 2. Ability to identify fall status of the Berg Balance Scale (BBS), Balance Evaluation 370 Systems Test (BESTest), Mini-BESTest and the Brief-BESTest $(n=66)$.

\begin{tabular}{cccccc}
\hline Balance test & AUC (SEM) & 95\% Cl & Cutoff point & $\begin{array}{c}\% \text { Sensitivity / } \\
\text { \% Specificity }\end{array}$ & LR $^{+/ \mathbf{L R}^{-}}$ \\
\hline BBS & $0.76(0.06)$ & $0.63 \rightarrow 0.88$ & 50.5 & $64 \% / 76 \%$ & $2.62 / 0.48$ \\
BESTest & $0.75(0.06)$ & $0.63 \rightarrow 0.87$ & 81 & $68 \% / 71 \%$ & $2.32 / 0.45$ \\
Mini-BESTest & $0.75(0.06)$ & $0.62 \rightarrow 0.87$ & 20.5 & $60 \% / 71 \%$ & $2.05 / 0.57$ \\
Brief-BESTest & $0.74(0.07)$ & $0.62 \rightarrow 0.87$ & 15.5 & $67 \% / 71 \%$ & $2.28 / 0.47$
\end{tabular}

371 Abbreviations: AUC, area under the curve; 95\% Cl, 95\% confidence interval; $\mathrm{LR}^{+}$, positive

372 likelihood ratio; LR-, negative likelihood ratio; BBS, Berg Balance Scale; BESTest.

373 
375 Figure 1. Scatterplots showing the relationship between the Activities-specific Balance 376 Confidence $(A B C)$ scale and the Berg Balance Scale (BBS), the Balance Evaluation Systems 377 Test (BESTest), the Mini-BESTest and the Brief-BESTest.
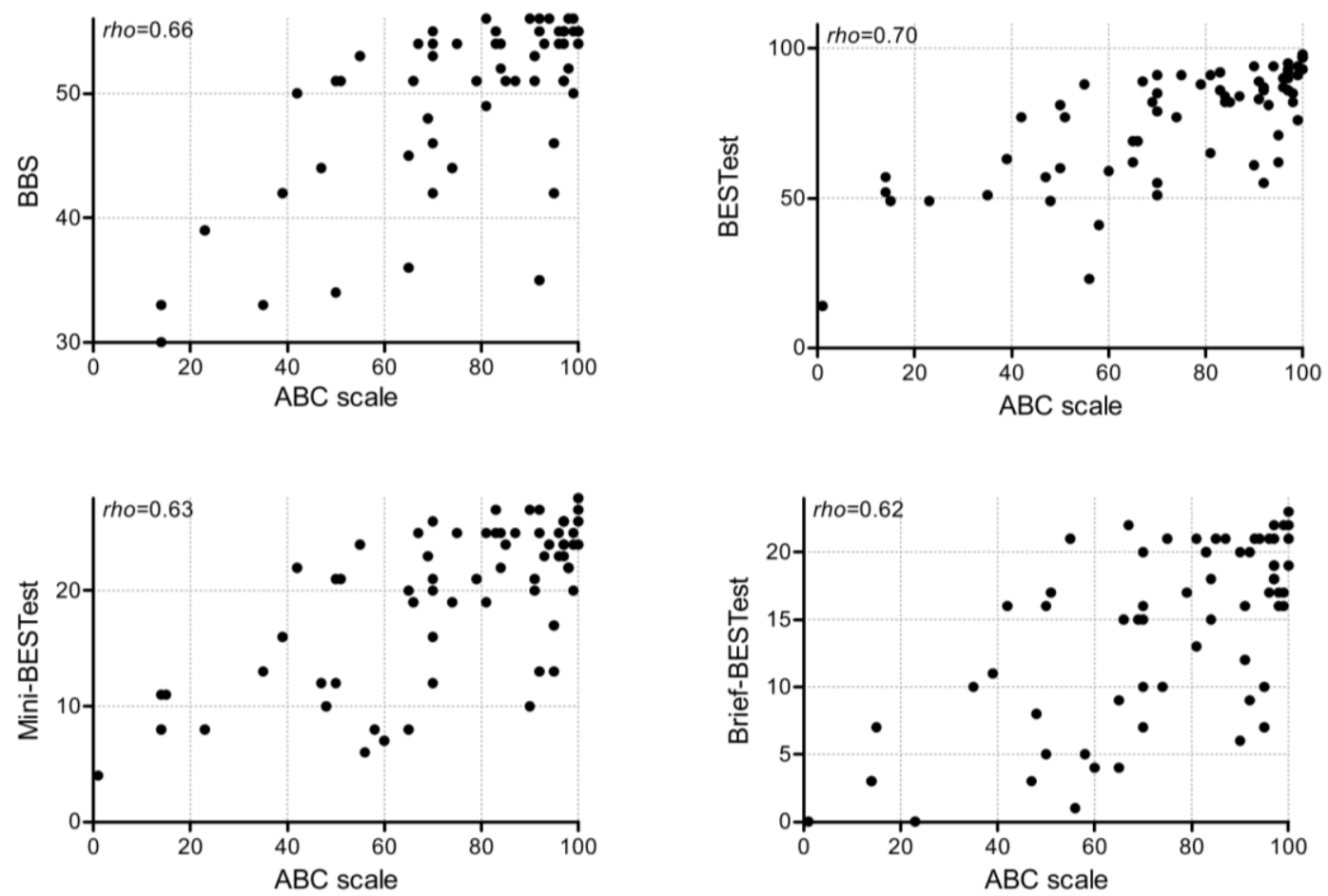
379 Figure 2. Receiver operator characteristics (ROC) of the Berg Balance Scale, the Balance 380 Evaluation Systems Test (BESTest), Mini-BESTest and the Brief-BESTest to differentiate 381 participants with and without a history of falls.

382

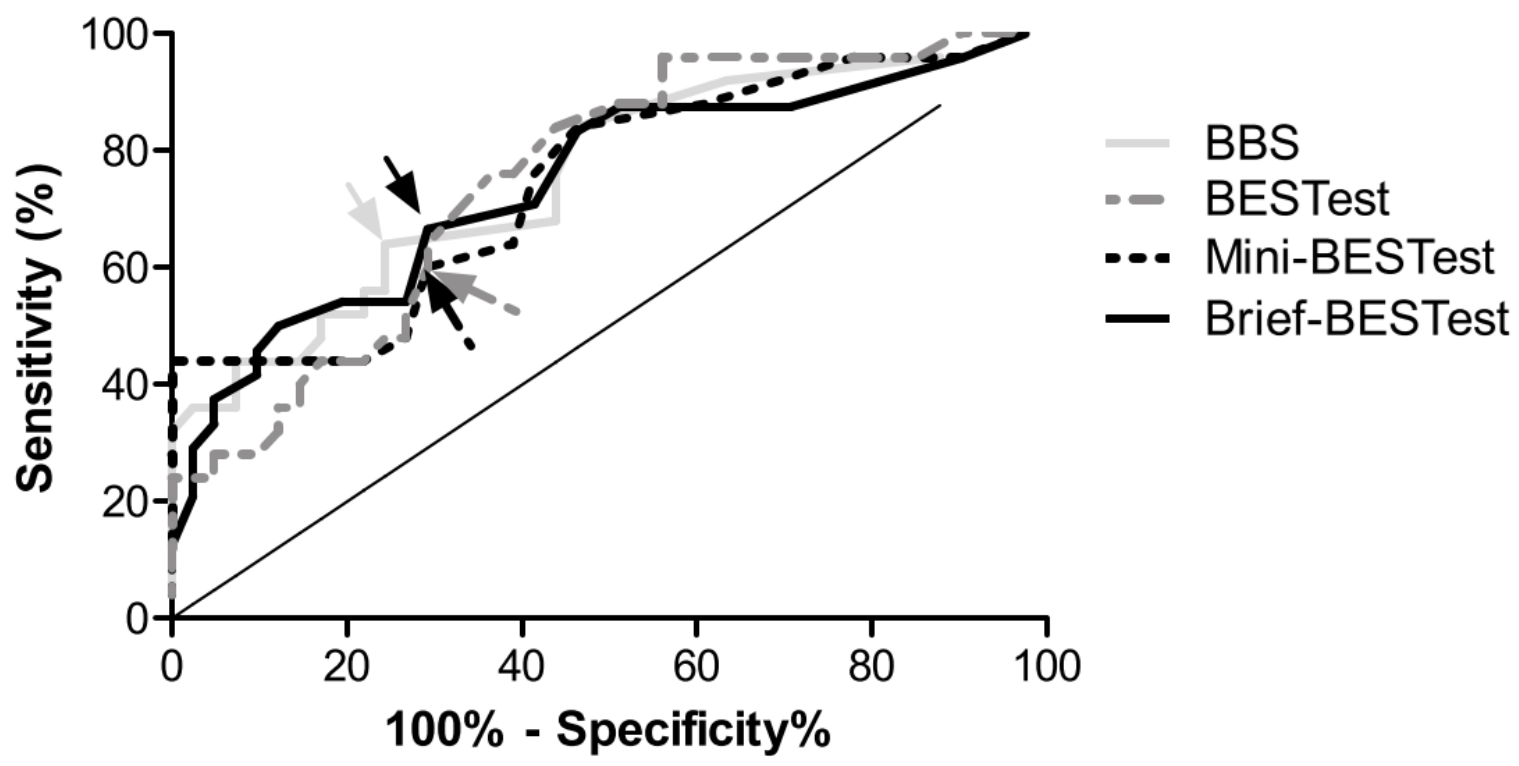

